\title{
Spanish Translations of Selected Abstracts
}

\author{
Translations by Graciela Zapata
}

\section{OF o no OF: El reto médico ante la escena de terrorismo químico}

Desde el ataque con Sarín de 1995, al subterráneo de Tokio, los ataques terroristas involucrando armas de destrucción masiva y otros químicos industriales, se han convertido en una preocupación de seguridad mundial. El triage y tratamiento medico en la escena, en este tipo de eventos es crucial para salvar tantas vidas como sea posible y minimizar los efectos dañinos del agente tóxico involucrado. Debido a la gran cantidad de químicos que pueden ser utilizados como armas terroristas, el reto para los Servicios Médicos de Emergencia (SME) es una combinación de: (1) reconocer que ha ocurrido un ataque químico terrorista (no convencional); e (2) identificar el agente tóxico seguido de un tratamiento con el antídoto adecuado. Lo anterior debe de realizarse tan rápido como sea posible, preferentemente en la escena. La decisión más valiosa en este punto, es determinar si se trata de un agente órganofosforado (OF) o un no OF, basado en los hallazgos clínicos observados por primeros respondientes previamente entrenados. Esta decisión es crucial, ya que sólo las intoxicaciones con $\mathrm{OF}$ cuentan con antídotos específicos, rápidamente disponibles y de acción rápida en la escena, como la atropina, preferentemente en presentación de auto inyección. La exposición a otros agentes (como químicos industriales, ej. Cloro, bromuro o amoniaco) deben de ser tratados en la escena, según su sintomatología, con medidas no específicas, como lo son, la descontaminación y el tratamiento de soporte, en vez de utilizar un antídoto. Este trabajo propone un algoritmo, como marco cognoscitivo, para los equipos médicos en la escena. Este algoritmo deberá ser parte del entrenamiento de los equipos médicos, en la preparación para ataques químicos terroristas, y el equipo debe de ser entrenado para usarlo, mediante simulacros. El implementar este tipo de pensamiento, mejorara los resultados médicos en este tipo de eventos.

Krivoy A, Layish I, Rotman E, Goldberg A, Yehezkelli Y: OP or Not OP: The medical challenge at the chemical terrorism scene. Prehosp Disast Med 2005;20(3):155-158.

El Uso de Observadores Entrenados como una Herramienta de Evaluación para un Ejercicio Multihospitalario de Bioterrorismo

Introducción: Las evidencias sugieren que los ejercicios regulares sobre desastres, tienen un efecto benéfico en subsecuentes simulacros o en desastres reales. El propósito de este reporte de caso, es la descripción de un ejercicio multihospitalario de bioterrorismo, utilizando observadores independientes con una plantilla de evaluación.
Métodos: El diseño del ejercicio incluyo la participación de 23 hospitales de la Comisión Conjunta de Acreditación de Organizaciones de Salud, 4 departamentos de salud, y un representativo de una agencia federal. El ejercicio fue evaluado por observadores entrenados, utilizando un protocolo de evaluación preparado independientemente.

Resultados: Todos los sitios del ejercicio identificaron correctamente el agente biológico involucrado y contestaron sin mucha dificultad a las preguntas realizadas después del ejercicio. La evaluación de los observadores independientes, en forma de un reporte post-acción, delato muchas de las limitaciones de la estructura del ejercicio.

Conclusiones: El contar con un grupo de observadores independientes probo proporcionar un valor agregado benéfico en la captura de información y datos subjetivos. Sin embargo, esta información se encontraba de una forma no apta para el análisis estadístico. Se requiere de un trabajo posterior para crear una herramienta de evaluación, que permita el análisis estadístico de forma, que los ejercicios puedan ser comparados y las mejoras juzgadas de manera objetiva.

Klein KR, Brandenburg DC, Atas JG, Maher A: Use of trained observers as an evaluation tool for a multi-hospital bioterrorism exercise. Prehosp Disast Med 2005;20(3): 159-163.

\section{Pronosticando el Trabajo Médico en Eventos Masivos: Modelo Predictivo Versus Revisión Retrospectiva}

Introducción: Los eventos masivos son dinámicos y retan los sistemas tradicionales de atención medica. Para mejorar el sistema de primeros auxilios en eventos masivos, este trabajo, presenta una evaluación de dos modelos que sirven para pronosticar, el número de pacientes a atender por los servicios médicos de urgencia.

Método: Se realizo una evaluación prospectiva de un evento masivo recurrente, comparando los pacientes pronosticados, y los traslados en ambulancia pronosticados, por un modelo predictivo, desarrollado por Arbon et al, y de una revisión retrospectiva, de siete años de información histórica del evento, descrita por Zeitz et al.

Resultados: El promedio de pacientes presentados (por 1,000 patrones) para este evento fue de 1.6, y el promedio de traslados al hospital (por 1,000 patrones) fue de 0.07. La revisión retrospectiva predijo cercanamente el número de atenciones. Ambos métodos, pronosticaron el número de pacientes presentados en una base diaria. La predicción probo ser más exacta en una base diaria, que con el método de Zeitz. 
Conclusión: El método de Rabón, es particularmente útil para eventos donde la información previa sobre la atención médica no existe, o es límitada. La revisión retrospectiva de la información generada por eventos específicos (método de Zeitz), considera las variables únicas e individuales, que pueden ocurrir de un evento a otro, y es más exacto para pronosticar la presentación de pacientes, cuando se tiene disponible la información. Ambos métodos, tienen el potencial de ser usados más frecuentemente, para una adecuada y eficiente planeación, de los recursos necesarios para los eventos.

Zeitz KM, Zeitz CJ, Arbon P: Forecasting medical work at mass-gathering events: Predictive model versus retrospective review. Prehosp Disast Med 2005;(20)3:164-168.

Análisis Prospectivo de la Capacidad de Expansión de Camas Hospitalarias en el caso de un Incidente con Víctimas Múltiples

Introducción: Las estrategias tradicionales para determinar la capacidad de expansión de camas hospitalarias, se ha basado en censos intersectoriales de los hospitales, los cuales subestiman la verdadera capacidad en un incidente con víctimas múltiples (IVM).

Objetivo: Determinar de una forma más exacta, la capacidad de expansión de camas hospitalarias en nuestro pais, usando evaluaciones realizadas a los supervisores de médicos y enfermeras, sobre la disposición de todos los pacientes en múltiples instalaciones.

Métodos: La evaluación fue realizada con jefas de enfermería, de turnos, tanto de día como de noche, de cada unidad y de cuatro hospitales diferentes, dicha evaluación incluyo a cada paciente según su ubicación supuesta a las 2 , 24 y 72 horas después de un IVM, incluyendo su traslado hipotético a una instalación de atención in-situ. Así mismo médicos de las dos instituciones académicas fueron incluidos para comparación. La edad, el género y el diagnostico de admisión, también fueron registrados para cada paciente.

Resultados: Se incluyeron un total de 1,741 evaluaciones de 788 pacientes, realizadas por 82 jefas de enfermería y 25 médicos, de 4 instituciones. Las jefas de enfermeras evaluaron como pacientes descartables, a aproximadamente un tercio de todos los pacientes a las 24 horas y aproximadamente un medio a las 72 horas; un cuarto de los pacientes fueron evaluados como transferibles a un centro de atención in-situ hipotético en ambos puntos de tiempo. Los médicos tendieron más que las jefas de enfermeria a mandar a pacientes a dicha instalación o a darlos de alta, pero tendieron menos a transferir pacientes fuera de las unidades de cuidados intensivos (UCI). La variación inter-institucional fue explicada por diferencias en la distribución de varios diagnósticos de los pacientes.

Conclusiones: Una gran proporción de pacientes puede ser dada de alta entre las 24 y 72 horas posteriores al IVM. Camas adicionales pueden ponerse a disposición si se cuen- ta con una instalación de atención in-situ. Tanto médicos como las jefas de enfermería deben de ser incluidos en el equipo que realiza la disposición de pacientes en un evento con víctimas múltiples.

Davis DP, Poste JC, Hicks T, Polk D, Rymer TE, Jacoby I: Prospective analysis of hospital bed surge capacity in the event of a mass-casualty incident. Prehosp Disast Med 2005;(20)3:169-176.

\section{Lesiones y Enfermedades Tratadas en el World Trade Center, Del 14 de Septiembre al 20 de Noviembre del 2001}

Introducción: En respuesta a los ataques terroristas del 11 de septiembre del 2001, en el World Trade Center (WTC), los Servicios de Salud Pública de Estados Unidos (USPHS), utilizaron Equipos Médicos de Asistencia en Desastres (DMATs) y las Corporaciones Comisionadas, para proveer asistencia medica in-situ, a todo aquel que se presentara. Los pacientes de los USPHS incluyeron trabajadores de rescate y recuperación, otros respondientes, y algunos miembros del público en general.

Objetivo: Se realizo un análisis descriptivo, de los expedientes, de los pacientes de los USPHS en el WTC, con el objetivo, de lograr un mejor entendimiento del impacto a corto plazo, en la seguridad y salud de los individuos ubicados cerca o en el WTC, entre el 14 de septiembre y el 20 de noviembre del 2001.

Métodos: Se revisaron los expedientes de tratamiento a pacientes, llenadas por cada visita de un paciente, a los módulos de atención de los USPHS, durante el periodo de 10 semanas.

Resultados: El número de pacientes fue 9,349, con el pico más alto de visitas durante la segunda semana (21 de septiembre al 27 de septiembre) y después tendiendo a disminuir. Más de un cuarto de las visitas se debieron a heridas traumáticas, sin incluir heridas en ojos $(n=2,716 ; 29 \%)$. Los problemas de tipo respiratorio conformaron arriba de un quinto de las atenciones $(\mathrm{n}=2,011 ; 22 \%)$. Los problemas de ojos comprendieron la tercera causa más frecuente de problemas $(n=1,120 ; 12 \%)$. Con respecto a la clasificación de triage, la mayoría de las visitas correspondieron a la categoria más baja de severidad ( $n=6,237 ; 67 \%)$.

Conclusión: Las visitas a los USPHS fueron probablemente limitadas a problemas menores, cuando se comparan contra los análisis de los reportes de los departamentos médicos y los casos de hospital, sin embargo, debido a la proximidad de los USPHS al desastre, el análisis de las formas realizadas por los USPHS, provee un panorama más amplio del impacto en la seguridad y salud de las personas cercanas o en el WTC.

Perritt KR, Boal WL: Injuries and illnesses treated at the World Trade Center, 14 September to 20 November 2001. Prehosp Disast Med 2005;20(3):177-183. 


\section{Una Encuesta sobre las Actitudes de los TUMS ante la Muerte}

Introducción: El propósito de este estudio fue, el recopilar información acerca de las actitudes de los TUMS (Técnicos en Urgencias Médicas) ante su entrenamiento, comodidad y roles cuando el paciente muere en la escena.

Métodos: Una muestra de 136 TUMS (de todos los niveles), de 14 diferentes estados, fueron cuestionados antes de la terminación de un programa de educación continua. Cerca del $40 \%(n=54)$ de los TUMS estaban cursando un programa de entrenamiento relacionado con la muerte, basado en el Currículo de Educación ante la Muerte y Entrenamiento de Crisis sm 1 , mientras un $60 \%(n=82)$ estaban cursando un programa de entrenamiento no relacionado con la muerte. Cada participante contesto preguntas acerca de sus actitudes, ante la muerte de un paciente en la escena, basados en la escala de cinco puntos de Likert. Los TUMS fueron comparados según su nivel de capacitación (TUM Básico, TUM Intermedio y TUM Avanzado), y según el tipo de programa educacional cursado (educación relacionada con la muerte y educación no relacionada con la muerte).

Resultados: La mayoría de los participantes (82\%), reportaron que las acciones de un TUM impactan el duelo de una familia desconsolada. Cerca de la mitad (54\%), reportaron, que el rol del TUM debería incluir notificar de la muerte a la familia. Sin embargo, tres cuartos (76\%), reportaron que no fueron entrenados adecuadamente en como notificar la muerte o en como ayudar a la familia con su dolor. Muchos (40\%), se sintieron incómodos notificando la muerte. Se encontraron diferencias entre los TUMS cursando programas de educación relacionados con la muerte y los que atendían a cursos no relacionados con la muerte. También fueron encontradas diferencias entre los diferentes niveles de TUMS (TUM Básico/ Intermedio contra los TUMS Avanzados).

Conclusión: Este estudio revelo nuevos conocimientos sobre las actitudes de los TUMS ante la muerte y el entrenamiento en relación a esta que reciben.

Smith-Cumberland, TL, Feldman, RH: A survey of EMT's attitudes toward death. Prebosp Disast Med 2005; 20(3):184-188.

\section{Problemas de Comunicación y de Protección a la Salud como resultado de una Emergencia por Inundación}

Objetivo: Identificar y describir los problemas clave, de comunicación y protección a la salud, alrededor de una emergencia por inundación.

Métodos: Se utilizo una encuesta telefónica para investigar la fuentes de información sobre salud y seguridad, la información recibida, y las respuestas ejecutadas por la población del pueblo de Feilding, en Nueva Zelanda, luego de una inundación mayor en febrero del 2004. Esto fue complementado, con entrevistas a informantes clave y un análisis del contenido de los periódicos.

Resultados: Se encuesto un total de 154 residentes adultos ( $76 \%$ de los cuales, fueron contactados exitosamente y eran elegibles). De los encuestados, el $79 \%$ reporto recibir información de salud y seguridad de las autoridades. Las fuentes principales fueron: radio (41\%), panfletos (23\%), y periódi$\cos (20 \%)$. El periódico regional tenía una cobertura extensa sobre la inundación, pero, "la salud", sólo era mencionada en 46, de 664 artículos relacionados con la inundación (7\%).

De aquellos que reportaron haber recibido información de las autoridades, la mayoría (89\%), la percibía como adecuada. Sin embargo, $18 \%$ buscaron información activamente por su cuenta (la mayoria acerca de disponibilidad y seguridad con el agua). En el periodo post-inundación, el $73 \%$ hirvió el agua; sin embargo, solo el $4 \%$, mantuvo el consumo de agua embotellada o hirvió el agua, por el periodo necesario de nueve semanas. Algunas formas de preparación para emergencias no cambiaron substancialmente después de la inundación, comparado contra cuatro meses antes (ej, tener un radio de pilas fue de un $62 \%$, a un $68 \%$ ). La percepción sobre riesgos a la salud alrededor de la inundación fue baja, el $59 \%$ estaba preocupado acerca del riesgo de contaminación del agua. Los reportes de problemas de salud relacionados con la inundación fueron raros, ya que solo dos personas de $154(1 \%)$, reportaron problemas de salud relacionados con la inundación.

Conclusiones: Este estudio encontró que la información sobre salud y seguridad fue exitosamente comunicada, y percibida como adecuada, por la población de este pueblo, afectado por la inundación. Sin embargo, queda un panorama abierto para mejorías futuras en las respuestas a emergencias, tanto para la población, como para las autoridades.

Wilson N, McIntyre M, McDonald M, Tanner H, Hart K, Tomlinson R, Thach T, Campion V, Lee D, Morrison F, Andersen E, Bibby S: Communication and health protections issues arising from a flooding emergency. Prehosp Disast Med 2005;20(3):193-196. 